\title{
BMJ Open An Application-based programme to reinforce and maintain lower salt intake (AppSalt) in schoolchildren and their families in China
}

Feng J He, ${ }^{1}$ Puhong Zhang, ${ }^{2,3}$ Rong Luo, ${ }^{2}$ Yuan $\mathrm{Li},{ }^{2,3}$ Fengge Chen, ${ }^{4}$ Yuhong Zhao, ${ }^{5}$ Wei Zhao, ${ }^{4}$ Daoxi Li, ${ }^{6}$ Hang Chen, ${ }^{6}$ Tianyong Wu, ${ }^{6}$ Jianyun Yao, ${ }^{7}$ Jinbao Li, ${ }^{7}$ Siyuan Zhou, ${ }^{7}$ Yu Liu, ${ }^{8}$ Xian Li, ${ }^{2,3}$ Changqiong Wang, ${ }^{1}$ Graham A MacGregor ${ }^{1}$

To cite: He FJ, Zhang $P$, Luo R, et al. An Applicationbased programme to reinforce and maintain lower salt intake (AppSalt) in schoolchildren and their families in China. BMJ Open 2019:9:e027793. doi:10.1136/ bmjopen-2018-027793

- Prepublication history for this paper is available online. To view these files, please visit the journal online (http://dx.doi. org/10.1136/bmjopen-2018027793).

FJH and PZ contributed equally.

Feng J He and Puhong Zhang are joint first authors

Received 7 November 2018

Revised 28 March 2019

Accepted 22 May 2019
Check for updates

(C) Author(s) (or their employer(s)) 2019. Re-use permitted under CC BY-NC. No commercial re-use. See rights and permissions. Published by BMJ.

For numbered affiliations see end of article.

Correspondence to Prof Feng J He; f.he@qmul.ac.uk and Prof Puhong Zhang; zpuhong@georgeinstitute.org.cn

\section{ABSTRACT}

Introduction Salt intake is very high in China, with $\approx 80 \%$ being added by the consumers. It is difficult to reduce salt in such settings. Our previous study (School-based Education programme to reduce Salt(School-EduSalt)) demonstrated that educating schoolchildren, who then instructed their families to reduce the amount of salt used at home, is effective in lowering salt intake in both children and adults. Our team also developed an app called 'KnowSalt', which could help individuals to estimate their salt intake and the major sources of salt in the diet. Building on School-EduSalt and KnowSalt, we propose to develop a new app (AppSalt) focusing on salt reduction through education, target setting, monitoring, evaluation, decision support and management to achieve a progressive lower salt intake for long term. To evaluate the effectiveness of the AppSalt programme, we will carry out a cluster randomised controlled trial.

Methods and analysis We will recruit 54 primary schools from urban and rural areas of three provinces in China. A total of 594 children aged 8-9 years and 1188 adult family members will be randomly selected for evaluation. After baseline assessment, schools will be randomly allocated to either the intervention or control group. Children in the intervention group will be taught, with support of AppSalt, about salt reduction and assigned homework to get the whole family involved in the activities to reduce salt consumption. The duration of the intervention is two school terms (ie, 1 year). The primary outcome is the difference between the intervention and control group in the change of salt intake as measured by 24-hour urinary sodium. Ethics and dissemination The study has been approved by Queen Mary Research Ethics Committee and Peking University Health Science Centre IRB. Results will be disseminated through presentations, publications and social media.

Trial registration number ChiCTR1800017553.

\section{INTRODUCTION}

Cardiovascular disease (CVD; ie, strokes, heart attacks and heart failure) is the leading cause of death and disability worldwide. Approximately $80 \%$ of CVD deaths occur in
Strengths and limitations of this study

- Our study will develop a new approach to achieving a sustainable progressive lower salt intake for long term.

- The study will use an innovative smartphone application through estimation of salt intake, education, target setting and monitoring, decision support and management to implement a salt reduction programme.

- The study covers a wide range of the population including children and adults in diverse settings, for example, rural and urban, northern, central and southern China.

- The results should generally be applicable to the whole Chinese population.

- The study will be carried out in China only; however, the method could potentially be adopted by many other developing countries where most of the salt in the diet is added by the consumers.

developing countries. ${ }^{1}$ Raised blood pressure (BP) is an important risk factor for CVD, accounting for $62 \%$ of strokes and $49 \%$ of coronary heart disease. $^{2}$ High salt intake is the major cause of raised BP. ${ }^{3}$ Randomised trials have demonstrated that a reduction in salt intake lowers BP in both hypertensive and normotensive individuals, in both adults and children. ${ }^{4-6}$ There is also compelling evidence that a lower salt intake is associated with a reduced risk of CVD and total mortality. ${ }^{78}$ Indeed, salt reduction is one of the most cost-effective measures to prevent hypertension and CVD. ${ }^{39}$ The WHO has recommended a $30 \%$ reduction in population salt intake by 2025 and also set a target of $<5 \mathrm{~g} / \mathrm{d}$ for all adults and lower levels for children. ${ }^{10}$ Many developed countries have started salt reduction initiatives. ${ }^{11}$ Salt intake has been successfully reduced in Finland and the UK, accompanied by falls in population 
BP and CVD mortality. ${ }^{12}$ Developing countries, however, are lagging behind.

China is the largest developing country with one-fifth of the world population. Salt intake in China is very high with an average of $12-14 \mathrm{~g} / \mathrm{d}$ in adults, ${ }^{13-15}$ and in some rural areas of northern China, salt intake could be as high as $18-20 \mathrm{~g} / \mathrm{d} .{ }^{16}$ Approximately $80 \%$ of the salt in the Chinese diet is added by the consumers during cooking or in sauces. ${ }^{17}$ It is extremely challenging to reduce salt intake in such settings due to the difficulty in changing individuals' dietary behaviours. Our previous study (School-based Education programme to reduce Salt (School-EduSalt)) has developed a novel and effective approach to reducing salt intake in northern China. ${ }^{13}$ In School-EduSalt, primary schoolchildren aged $\approx 10$ years were educated about the harmful effects of salt on health and how to reduce intake during the school's usual health education lessons. Children then instructed their families to reduce salt. The results showed that, over one school term $(\approx 3.5$ months), salt intake was reduced by $\approx 25 \%$ in both children and adults as measured by 24-hour urinary sodium excretion. The reduction in salt intake led to a significant fall in systolic BP of $2.3 \mathrm{~mm} \mathrm{Hg}$ in adults. ${ }^{13} \mathrm{It}$ was estimated that, if School-EduSalt was implemented and sustained, it would prevent $\approx 400000$ stroke and heart attack events per year in China.

Previous studies have shown that salt reduction achieved by dietary advice attenuates over time and maintaining a lower salt intake long-term remains a challenge. ${ }^{4}$ The School-EduSalt was successful over one school term of $\approx 3.5$ months. The question is how to continue and reinforce the salt reduction education to achieve a sustainable, progressive lower intake to achieve the WHO's target.

Mobile technology is increasingly used in health education. ${ }^{18}$ Recent studies have suggested that game-based intervention has great potential in changing behaviours due to the popularity of gaming. ${ }^{19}$ China has the largest and fastest growing mobile internet population with 802 million people using the internet and mobile phone penetration reached $98.3 \%$ of the population by June 2018. ${ }^{20}$ Mobile-based decision support system has been shown to be effective in increasing medication use in individuals with high CVD risk in rural China. ${ }^{21}$ Our team has recently developed a smartphone application (KnowSalt), which can be used by individuals to estimate their salt intake and the major sources of salt in the diet. The methodology underpinning KnowSalt application had been validated in a pilot study in primary schoolchildren and their families. ${ }^{16}$

Our proposed study building on School-EduSalt and KnowSalt app will develop an innovative smartphone application focusing on salt reduction (AppSalt) through functional modules of education, evaluation, target setting and monitoring, decision support and management, with the aim of reinforcing School-EduSalt to achieve a progressive lower salt intake for long term. To evaluate the effectiveness and cost-effectiveness of the AppSalt programme, we will carry out a cluster randomised trial.

\section{METHODS AND ANALYSIS}

The project consists of two phases: (1) developing AppSalt platform and (2) carrying out a cluster randomised controlled trial (RCT).

\section{Phase 1-development of AppSalt platform}

AppSalt platform comprises a smartphone application named AppSalt (family end) and a WeChat mini app (teacher end). The AppSalt consists of four core procedures (ie, education, evaluation, decision support and reminding) and three supportive modules (ie, Competition \& Award, Supervision \& Communication and SaltSwitch) (figure 1).

\section{Core procedures}

1. 'Education' is to educate the families about the harmful effects of salt on health and how to reduce salt intake. Nine lessons are developed, each starting with a 10-min lecture on salt reduction, followed by a few questions to test whether the families have understood the content. At the same time, the questions and answers will re-enforce the key messages. Following each lesson, the families are required to complete a practical session, which is designed to help the families to prepare foods with reduced salt at home and choose lower-salt foods when shopping.

2. 'Evaluation' is to estimate salt intake and the main sources of salt for every member of the family. After completing a simple questionnaire, the family will be provided with the information on the top three contributors of salt in their diet. This will help the family make a decision on how to effectively reduce salt intake. Evaluation of the top three contributors (ie, top three sources of dietary salt) will be based on a 7-day salt surveillance method. Participants will record frequency of dining out, consumption of processed foods and the amount of salt added during cooking. The added salt will be assessed by weighing salt, soy sauce and other primary salty condiments on the first and last day of the evaluation period. ${ }^{16}$

3. 'Decision support' will set target for total salt intake and specific targets for top three contributors. Lower-salt sample recipes and specific measures, for example, reducing the amount of salt used during cooking by $50 \%$, will be recommended. The recommended measures will be customised as an action plan.

4. 'Reminding' will remind the family how far their salt intakes are away from the targets set and highlight further action plans.

\section{Supportive modules}

1. 'Supervision \& Communication' module: The teachers and researchers will use the function 'Supervision' to announce instructions, newsletters and school canteen 


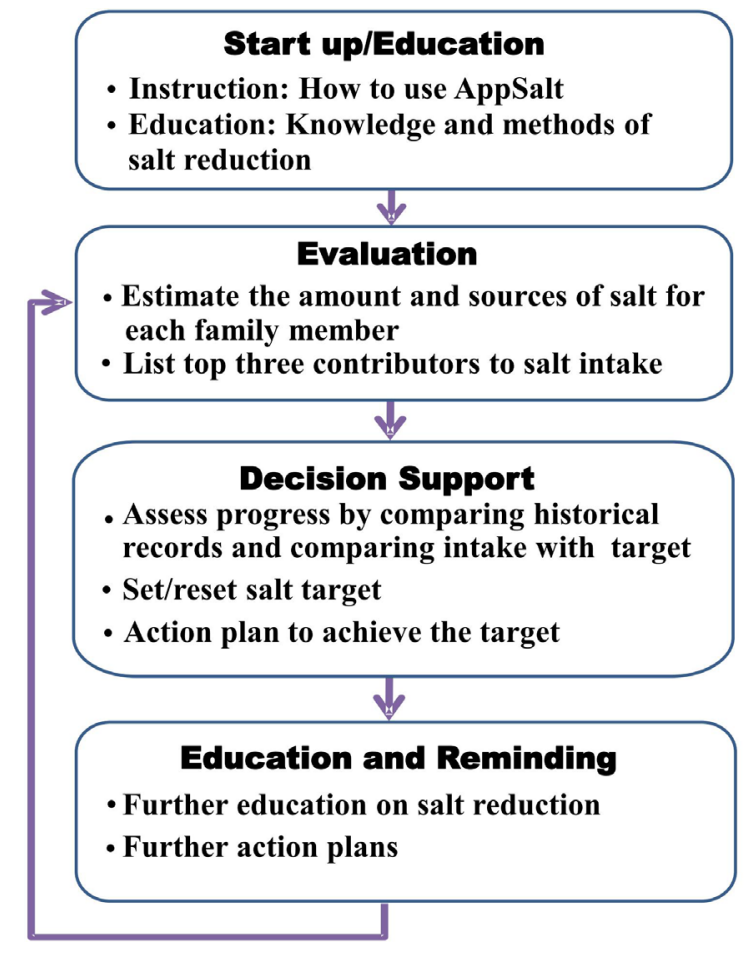

Figure 1 AppSalt core procedures and modules.

menus, as well as providing answers to any queries. The participants will use the function 'Communication' to ask any questions and also exchange experience and knowledge with other participants.

2. The 'SaltSwitch' module will help the participants to choose prepackaged foods with lower salt. 'SaltSwitch', as a subfunction of 'FoodSwitch' ${ }^{22}$ integrated into the AppSalt, has a database of over 50000 prepackaged foods collected by The George Institute (TGI) China. By scanning the barcode of the prepackaged foods, the 'SaltSwitch' will provide information on salt/sodium content and compare it with other similar products based on products' labelling.

3. The 'Competition \& Award' module will help the participants to find out their own ranking position assessed by salt intake level and the extent of salt reduction among all participants (NB: only their own ranking will be displayed, and other participants' ranking will not be revealed to them). The family with the lowest salt intake and the family who achieve the greatest reduction in salt intake will be the winners of competition. They will be awarded an honorary title like " Salt Reduction Pacesetter' and get a small gift.

AppSalt will be installed in the smartphone of adult family members. Children are only allowed to use AppSalt accompanied by their adult family members to complete AppSalt core procedures as required. New features with a variety of presentation styles on salt reduction education will be added to keep the programme attractive to the participants.

The school teachers will use the WeChat mini app (teacher end) to send messages to the families, provide timely feedback to any queries and check families'

\section{Supervision and Communication}

- Give instruction/request/reminder by teacher

- Share experience among peers

SaltSwitch
- Provide information on salt/sodium
content
- Choose prepackaged foods with
lower salt

\section{Competition and Award}

- Find out ranking position of salt intake compared with others

- Assess achievement

- Receive award from teacher for completion winners progress and ensure all families complete the procedures in time. The teachers will also report any issues to the site managers and the research team where appropriate.

\section{Phase 2-cluster RCT}

To test the effectiveness and cost-effectiveness of the AppSalt programme, we will carry out a cluster RCT (figure 2).

\section{Study setting and participants}

Considering the geographical location, economic level and dietary habit, our study will be undertaken in selected primary schools from urban and rural areas of three provinces in northern (Hebei), central (Sichuan) and southern (Hunan) China.

To recruit schools, we will first contact the local education authority through local Health/Education Bureau or Centre for Disease Control to gain their opinion, support and approval. We will then contact schools' headteachers to request their participation in the study. In each school, there are six grades with age ranging from 7 to 12 years, and our study will be carried out in grade 3 (age 8-9 years). Head- teacher will choose one class whose teacher in charge is willing to collaborate with the researchers. From each class, we will randomly select 11 children and 22 adult family members (ie, one child and two adults per family) for evaluation. The inclusion criteria are (1) children and their adult family members have to eat homemade meals at least four times per week; (2) one of the adults in the family has a mobile device with access to Internet; (3) if more than two adults in one family agree to take part in the evaluation, we will select two in the order of grandparents, parents, uncles 


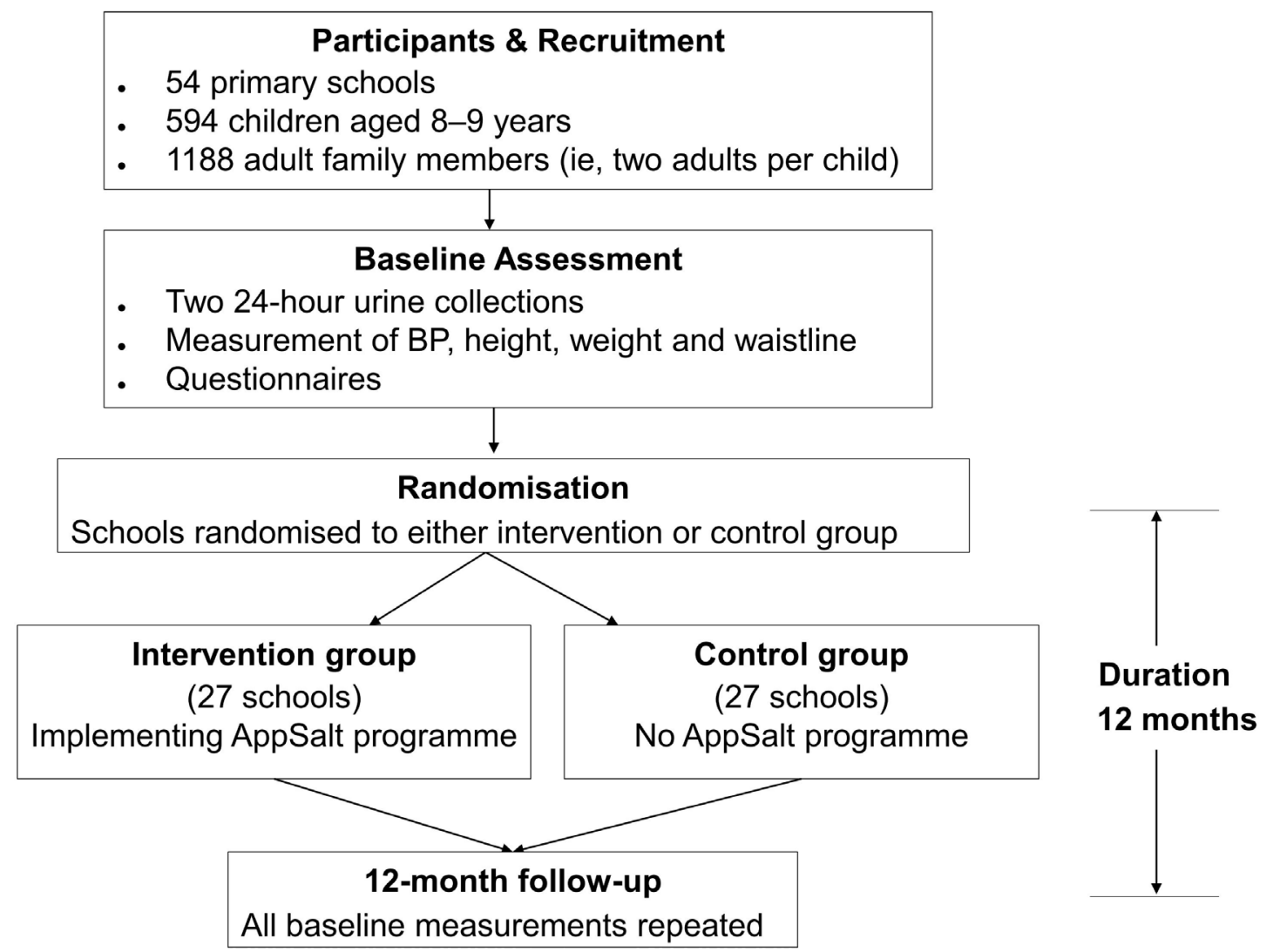

Figure 2 AppSalt trial design. BP, blood pressure.

and aunties; (4) participants have been local residents for over 6 months, without moving plan within 24 months. We will exclude the individuals who cannot or refuse to collect 24-hour urine.

\section{Randomisation}

Schools (clusters) will be randomly assigned (1:1) to either the intervention or the control group. Randomisation will be stratified by the location of schools (ie, south or north, urban or rural) and the size of the class. The randomisation will be carried out using computer-generated random number by an independent statistician who is blind to the identity of the schools. The randomisation will take place after baseline data collection has completed. Therefore, the participants, school-teachers and local investigators who undertake recruitment and data collection, will be unaware of the allocation until the point prior to the commencement of the intervention.

\section{Intervention}

Our goal is to lower salt intake (measured by 24-hour urinary sodium) by a minimum of $15 \% \quad(\approx 1.5 \mathrm{~g}$ salt $)$ for both children and their families. To achieve this goal, a strategy towards a $50 \%$ reduction should be implemented. To make the decision-support algorithm simple in the AppSalt, we will set targets for the top three contributors to salt intake, such as cooking salt. Targets and priority actions will be individualised depending on the sources of salt in the individual's diet, which will be provided by AppSalt. During the education lessons delivered through AppSalt, the participants will be taught the methods on how to effectively reduce salt intake, for example, reducing the amount of salt, soy sauce and bean paste used during cooking. Garlic, ginger and herbs are recommended for enhancing food flavour. We also recommend replacing pickles with fresh vegetables and replacing salted eggs and peanuts with unsalted ones.

In the intervention group, the AppSalt intervention package will be delivered to all children and their families in the whole class, despite only 11 children and their families being randomly selected for evaluation. The adult family members will be authorised to install and operate the AppSalt programme, while children will be assigned homework to get the whole family to reduce salt with the support of AppSalt. Children's homework will include (1) helping the family to set salt reduction target and reminding the whole family of this target on a regular basis; (2) getting the whole family involved in salt reduction activities and persuading the person who does the cooking to reduce the amount of salt used during food preparations at home; (3) working with their family members to complete the AppSalt core procedures, including education, evaluation, target setting and monitoring. In addition to the online lessons, school teachers will organise three face-to-face seminars for both children and adults during the 1-year intervention period. This will provide an opportunity for the participants to share experiences and discuss challenges and solutions. These 
seminars will be integrated into the schools' routine parent meetings.

Trained teachers will provide guidance on AppSalt utilisation, assign and check children's homework including monitoring the progress of salt reduction. They will also create salt-reduction environment, for example, putting up posters in classroom, campus and canteens. The AppSalt will be activated at the beginning of the intervention for all families in the intervention group. Throughout the study, participants will receive salt reduction messages and newsletters via AppSalt on a monthly basis. Participants will be encouraged to use AppSalt and communicate with the teachers and other families to share experiences. The mandatory tasks for the participants include completing the online lessons and the related questions and answers at baseline and every month thereafter and completing the core procedures, that is, salt intake estimation, target setting at baseline and every 3 months thereafter. In addition to the mandatory tasks, the participants are encouraged to repeat the core procedures as often as they wish in order to monitor their progress and achieve progressive reductions in salt intake. Based on the progress monitored from the WeChat mini application, the teachers will remind children that they should complete their homework, which is to help the family complete the AppSalt procedures in time and to instruct the family members to reduce salt to the target.

The records from AppSalt, for example, salt intake and how far the intake is different from the target set, will be used to monitor compliance. During follow-up, the participant who fails to achieve the salt reduction target will be sent a specially designed message via AppSalt and a letter to the child through his/her teacher to remind the family of the salt reduction target.

The intervention duration is two school terms, that is, 1 year. Children in the control group will carry on with their usual health education lessons as in the curriculum. After the trial is completed, we will introduce the AppSalt to all schoolchildren and their families including those in the control group.

\section{Sample size calculation}

Based on the School-EduSalt trial, ${ }^{13}$ assuming an SD of 24-hour sodium excretion is $85 \mathrm{mmol} / \mathrm{d}$, and intraclass correlation coefficient is 0.05 , we estimate that a sample size of 594 children from 54 schools (18 primary schools from each province in both urban and rural area and 11 children per school) would provide a power of $80 \%$ (with a two-sided alpha $=0.05$ ) to detect a difference in mean 24-hour urinary sodium $\geq 26 \mathrm{mmol} / \mathrm{d}$ ( $1.5 \mathrm{~g} / \mathrm{d}$ salt) between intervention and control groups, allowing for a $15 \%$ drop-out rate of participants. We aim to recruit two adults per family; therefore, 1188 adults will be recruited into the study for evaluation.

\section{Outcome measures}

The primary outcome is the difference between the intervention and control groups in the change of salt intake as measured by 24-hour urinary sodium from baseline to the end of the trial. The secondary outcome is the difference between the two groups in the change of systolic BP for adults.

\section{Outcome assessments}

All outcome measurements and assessments will be carried out at baseline and at the end of the trial in exactly the same way in all schools for all participants, irrespective of their assignment to intervention or control group.

Two consecutive 24-hour urine collections will be made at each time point, using the stringent protocol which we developed in the School-EduSalt study. ${ }^{13}$ Although there are other more convenient methods for assessing salt intake, for example, a food diary, they are not reliable, and the most accurate method is 24-hour urine collection. ${ }^{23}$ Our previous studies have shown that it is entirely feasible to collect 24-hour urine in both children and adults. ${ }^{13}$ The participants will be carefully instructed on how to accurately collect 24-hour urine by trained research staff. On the first day, the participants will be asked to come to the schools. The researchers will ask the participants to empty bladder and discard the urine. The researchers will record the start time and date of the 24-hour urine collection. They will then give the participants the collection equipment including containers and collection aids such as carrier bags. The participants will be instructed to collect all subsequent urine voids over the next 24-hour period. On the second day at the same time, the participants will be asked to bring the urine collection bottles back to the schools, and they will be asked to pass the last urine into the container. The researchers will record the finish time of the first 24-hour urine collection. The researchers will then give the participants the collection equipment for the second 24-hour urine collection and repeat the process. During the 24-hour urine collection period, the participants will be asked to take spare urine containers with them when they go to school or work. Spare collection equipment will also be available in the schools, in case children forget to bring them. In the case that the participant misses one or more urine voids or spillage occurs, the participant will be asked to do a further 24-hour urine collection. The 24-hour urine collections will be made either at weekdays or weekends. For each participant, the 24-hour urines will be collected on the same days of the week for baseline and follow-up at the end of the trial.

We will measure urine volume, sodium, potassium and creatinine. Ion-selective electrode method will be used for sodium and potassium analysis and enzymatic method for creatinine assay. Twenty-four-hour urinary creatinine together with urine volume will be used to determine if the collection is likely to be complete. The biochemist who performs the measurements of urinary electrolytes will not be told to which group the participant is allocated.

In addition to 24-hour urine collection, we will measure $\mathrm{BP}$ and heart rate using a validated automatic machine with the appropriate size of cuff. Three readings will be 
taken in the right arm at 1- to 2-min intervals at sitting position after the participant has had 10-min rest in a quiet room. Body weight, height and waist circumference will also be measured. Survey on Knowledge, Attitude and Practice (KAP) related to salt will be completed via questionnaire.

\section{Data collection, management and analysis Data collection}

Our study will have two major data outputs. One is the 'App Data' generated automatically when the AppSalt platform is used by the families in intervention group to support salt reduction. The App Data includes information on demographic characteristics, eating habits, amount and sources of salt in the diet, as well as information (or App logs) on the use of AppSalt. The other data output is the 'RCT Data', which is generated by both intervention and control groups during the RCT. The RCT Data will be collected through a specially designed mobile device-based electronic data capture system (mEDC) by well-trained field investigators. The mEDC has been validated and widely used in other clinical trials ${ }^{24}$ with more advantages on process and quality control compared with traditional EDC. The RCT Data includes information on demographics, social-economic information, KAP, measurement of height, weight, waist circumference, BP, heart rate, 24-hour urine volume and electrolytes, as well as information on costs for all the components of intervention conducted through the AppSalt programme.

\section{Data management}

All cleaned and locked datasets together with the study design, questionnaire, code list and definition of database and variables will be stored with a unique ID number attached but no personal identifiers, in TGI, following an established standard operating procedure for data security. To guarantee the data security, the mobile app developer (ie, the Information Technology (IT) team at Beihang University) must follow the 'Mobile Application Information Service Regulation' issued by the Cyberspace Administration of China in 2016. Although personal data are accessible to app developer, disclosure of such information is prohibited. In addition to the development and maintenance of the AppSalt and mEDC, IT team will also provide data management service during the study to guarantee the safety, integrity and proper use of the data collected through AppSalt and mEDC.

\section{Statistical analysis}

Data analyses will be performed according to the intention-to-treat approach. The effect of the intervention on the outcomes will be tested using linear mixed models with participants nested within family units and families nested within schools. We will include group (intervention, control), time (baseline, end of trial) and timexgroupinteraction, with the timexgroupinteraction term indicating differential change by group from baseline to the end of the trial. We will adjust for the stratification variables at randomization and potential confounding variables. Various sensitivity analyses will be carried out to examine the robustness of the conclusions of the primary analysis. Results will be reported as mean, SD, SE, and 95\% CI where appropriate. All analyses will be two sided, and $\mathrm{p}<0.05$ is considered significant. SAS will be used for the analyses.

\section{Economic evaluation and process evaluation}

Economic evaluations will be carried out from health sector perspective to compare the m-Health strategy with usual care, and it will entail two components: a trialbased economic evaluation and a modelled economic evaluation of long-term costs and outcomes. Intervention costs will include those in running the programme but exclude any research and development costs. The trialbased economic evaluation will be assessed initially in terms of incremental cost per unit reduction in salt intake and systolic BP. The modelled economic evaluation with discounting will examine the cost, survival, quality of life over lifetime, via capturing various health states (including death and CVD events) to estimate incremental cost per life year saved and cost per Quality-Adjusted Life Year gained. The transition probabilities across health states and costs attached to various health states will be based on literature and the long-term effects of the reduction in salt intake or systolic BP will be derived from the trial findings and/or literature of disease progression. Sensitivity analyses will be carried out to estimate uncertainty about the primary findings associated with varying key parameters.

Mixed-method process evaluations will be conducted during and at the end of the study using data from AppSalt, key informant interviews with researchers and teachers and focus groups interviews with participants. This will help understand the barriers and facilitators of the intervention as well as the acceptance and effect of the AppSalt programme.

\section{Project status and timelines}

Recruitment of schools and participants started in September 2018. Baseline assessments were carried out between September and December 2018. As the intervention duration is 1 year, the final follow-up assessments will be carried out between September and December 2019.

\section{Expected outcome and potential impact}

The study will provide a novel, feasible and effective approach to achieving a sustainable reduction in salt intake in both children and adults. The use of AppSalt platform in delivering health education lessons is particularly advantageous over the traditional school education method in terms of reducing the burden on teaching staff. Furthermore, our study covers a wide range of the population (children and adults, rural and urban, north and south), the results should therefore be generally applicable to the whole Chinese population. If the programme is implemented and sustained across China, it will reduce 
population salt intake and thereby prevent hundreds of thousands of strokes, heart attacks and heart failure each year, and lead to major cost-savings to individuals, their families and the health service.

Although our study will be carried out in China, the AppSalt programme could potentially be adopted by many other countries. Additionally, our model on salt reduction could possibly be adapted for other dietary and lifestyle changes to prevent CVD and other non-communicable diseases, which will have major public health implications.

\section{Patient and public involvement}

During the development of the study protocol, we had two round-table meetings with six head teachers from three different cities and several telephone meetings with teachers and children's parents to gain their opinion, particularly on the feasibility of carrying out the study in school settings and how to incorporate the intervention programme into the school curriculum. During the study, school teachers will assign and check children's homework. Both teachers and head teachers will be informed of the study progress by monthly communication via newsletters, WeChat and website. On completion of the study, we will disseminate the results to head teachers, teachers, children and their parents. We will also discuss with them and other stakeholders on how to translate research findings into practice.

\section{ETHICS AND DISSEMINATION}

Written consent will be obtained from all participants according to well-established practices. For children, participant assent and parental written consent will be obtained. All participants will be free to discontinue their participation at any time with no explanation required.

The findings of this study will be disseminated widely through conference presentations, peer-reviewed publications, press release and social media. Furthermore, the results will be disseminated worldwide through World Action on Salt and Health, ${ }^{25}$ which is a global non-profit organisation with 600 members from 100 countries with the mission to improve the health of populations by reducing salt intake worldwide.

\section{Author affiliations}

${ }^{1}$ Wolfson Institute of Preventive Medicine, Barts and The London Scho ol of Medicine \& Dentistry, Queen Mary University of London, London, UK

${ }^{2}$ The George Institute for Global Health at Peking University Health Science Center, Beijing, China

${ }^{3}$ Faculty of Medicine, University of New South Wales, Sydney, Australia

${ }^{4}$ Shijiazhuang Center for Disease Control and Prevention, Shijiazhuang, China

${ }^{5}$ Changan Center for Disease Control and Prevention, Shijiazhuang, China

${ }^{6}$ Luzhou Center for Disease Control and Prevention, Luzhou, China

${ }^{7}$ Yueyang Center for Disease Control and Prevention, Yueyang, China

${ }^{8}$ School of Computing, Beihang University, Beijing, China

Acknowledgements The authors would like to thank the head teachers, teachers and parents who shared their opinion on the development of the intervention programme.
Contributors FJH and PZ conceived the project and contribute equally to the work. FJH, PZ, YLi and RL designed the study and oversaw the conceptualisation and development of the app. PZ, YLi, RL, FC, YZ, WZ, DL, HC, TW, JY, JL and SZ facilitates Patient and Public Involvement and were responsible for setting up the study in each site. YLiu and RL are leading the development and maintenance of the app. $\mathrm{XL}$ and $\mathrm{CW}$ contributed to sample size calculation and analysis plan. All authors contributed to the development of the intervention and evaluation. FJH wrote the first draft of the manuscript, and PZ, YLi, RL, CW and GAM revised the draft. All authors contributed to the refinement of the study protocol and approved the final manuscript.

Funding This work is supported by the National Institute of Health Research using Official Development Assistance (ODA) funding (16/136/77).

Disclaimer This research was commissioned by the National Institute for Health Research (NIHR) (NIHR Global Health Research Unit Action on Salt China (ASC) at Queen Mary University of London) using Official Development Assistance (ODA) funding (16/136/77). The views expressed in this publication are those of the authors and not necessarily those of the NIHR or the Department of Health and Social Care.

Competing interests None declared.

Patient consent for publication Not required.

Ethics approval The study has been approved by Queen Mary Research Ethics Committee and Peking University Health Science Centre Institutional Review Board.

Provenance and peer review Not commissioned; externally peer reviewed.

Open access This is an open access article distributed in accordance with the Creative Commons Attribution Non Commercial (CC BY-NC 4.0) license, which permits others to distribute, remix, adapt, build upon this work non-commercially, and license their derivative works on different terms, provided the original work is properly cited, appropriate credit is given, any changes made indicated, and the use is non-commercial. See: http://creativecommons.org/licenses/by-nc/4.0/.

\section{REFERENCES}

1. Lawes CM, Vander Hoorn S, Rodgers A. Global burden of bloodpressure-related disease, 2001. Lancet 2008;371:1513-8.

2. The world health report 2002 - Reducing Risks, Promoting Healthy Life. Geneva, Switzerland: World Health Organisation, 2002. www. who.int/whr/2002

3. He FJ, MacGregor GA. Reducing population salt intake worldwide: from evidence to implementation. Prog Cardiovasc Dis 2010;52:363-82.

4. He FJ, Li J, Macgregor GA. Effect of longer term modest salt reduction on blood pressure: Cochrane systematic review and metaanalysis of randomised trials. BMJ 2013;346:f1325.

5. He FJ, MacGregor GA. Importance of salt in determining blood pressure in children: meta-analysis of controlled trials. Hypertension 2006;48:861-9.

6. Aburto NJ, Ziolkovska A, Hooper L, et al. Effect of lower sodium intake on health: systematic review and meta-analyses. BMJ 2013;346:f1326.

7. He FJ, MacGregor GA. Salt reduction lowers cardiovascular risk: meta-analysis of outcome trials. Lancet 2011;378:380-2.

8. He FJ, Campbell NRC, Ma Y, et al. Errors in estimating usual sodium intake by the Kawasaki formula alter its relationship with mortality: implications for public health. Int J Epidemiol 2018;47:1784-95.

9. Asaria P, Chisholm D, Mathers C, et al. Chronic disease prevention: health effects and financial costs of strategies to reduce salt intake and control tobacco use. Lancet 2007;370:2044-53.

10. WHO issues new guidance on dietary salt and potassium. 2013. http://www.who.int/mediacentre/news/notes/2013/salt_potassium_ 20130131/en/ [Accessed 30 Jan 2017].

11. Trieu K, Neal B, Hawkes $C$, et al. Salt Reduction Initiatives around the World - A Systematic Review of Progress towards the Global Target. PLoS One 2015;10:e0130247.

12. He FJ, Pombo-Rodrigues S, Macgregor GA. Salt reduction in England from 2003 to 2011: its relationship to blood pressure, stroke and ischaemic heart disease mortality. BMJ Open 2014;4:e004549.

13. He FJ, Wu Y, Feng XX, et al. School based education programme to reduce salt intake in children and their families (School-EduSalt): cluster randomised controlled trial. BMJ 2015;350:h770.

14. Zhou BF, Stamler J, Dennis B, et al. Nutrient intakes of middle-aged men and women in China, Japan, United Kingdom, and United States in the late 1990s: the INTERMAP study. J Hum Hypertens 2003;17:623-30. 
15. Zhang JY, Yan LX, Tang JL, et al. Estimating daily salt intake based on $24 \mathrm{~h}$ urinary sodium excretion in adults aged 18-69 years in Shandong, China. BMJ Open 2014;4:e005089.

16. Zhang L, Zhao F, Zhang P, et al. A pilot study to validate a standardized one-week salt estimation method evaluating salt intake and its sources for family members in China. Nutrients 2015;7:751-63.

17. Anderson CA, Appel LJ, Okuda N, et al. Dietary sources of sodium in China, Japan, the United Kingdom, and the United States, women and men aged 40 to 59 years: the INTERMAP study. J Am Diet Assoc 2010;110:736-45.

18. Burke LE, Ma J, Azar KM, et al. Current science on consumer use of mobile health for cardiovascular disease prevention: a scientific statement from the American Heart Association. Circulation 2015;132:1157-213.

19. Payne HE, Moxley VB, MacDonald E. Health Behavior Theory in Physical Activity Game Apps: A Content Analysis. JMIR Serious Games 2015;3:e4.
20. CNNIC. The 42nd Statistical Report on the Development of China's Internet Network. 2018. https://cnnic.net.cn/gywm/xwzx/rdxw/ 20172017_7047/201808/t20180820_70486.htm [Accessed 1 Nov 2018].

21. Tian M, Ajay VS, Dunzhu D, et al. A Cluster-Randomized, Controlled Trial of a Simplified Multifaceted Management Program for Individuals at High Cardiovascular Risk (SimCard Trial) in Rural Tibet, China, and Haryana, India. Circulation 2015;132:815-24.

22. Dunford E, Trevena H, Goodsell C, et al. FoodSwitch: a mobile phone app to enable consumers to make healthier food choices and crowdsourcing of national food composition data. JMIR Mhealth Uhealth 2014;2:e37.

23. McLean RM. Measuring population sodium intake: a review of methods. Nutrients 2014;6:4651-62.

24. Zhang J, Sun L, Liu Y, et al. Mobile Device-Based Electronic Data Capture System Used in a Clinical Randomized Controlled Trial: Advantages and Challenges. J Med Internet Res 2017;19:e66.

25. World Action on Salt and Health. http://www.worldactiononsalt.com 